\title{
A Novel Interference Detection Method of STAP Based on Simplified TT Transform
}

\author{
Qiang Wang, Yongshun Zhang, Hanwei Liu, and Yiduo Guo \\ Air and Missile Defense College, Air Force Engineering University, Xian, Shaanxi 710051, China \\ Correspondence should be addressed to Qiang Wang; 1019611183@qq.com
}

Received 20 June 2017; Accepted 26 October 2017; Published 21 November 2017

Academic Editor: Fazal M. Mahomed

Copyright (C) 2017 Qiang Wang et al. This is an open access article distributed under the Creative Commons Attribution License, which permits unrestricted use, distribution, and reproduction in any medium, provided the original work is properly cited.

\begin{abstract}
Training samples contaminated by target-like signals is one of the major reasons for inhomogeneous clutter environment. In such environment, clutter covariance matrix in STAP (space-time adaptive processing) is estimated inaccurately, which finally leads to detection performance reduction. In terms of this problem, a STAP interference detection method based on simplified TT (timetime) transform is proposed in this letter. Considering the sparse physical property of clutter in the space-time plane, data on each range cell is first converted into a discrete slow time series. Then, the expression of simplified TT transform about sample data is derived step by step. Thirdly, the energy of each training sample is focalized and extracted by simplified TT transform from energy-variant difference between the unpolluted and polluted stage, and the physical significance of discarding the contaminated samples is analyzed. Lastly, the contaminated samples are picked out in light of the simplified TT transform-spectrum difference. The result on Monte Carlo simulation indicates that when training samples are contaminated by large power target-like signals, the proposed method is more effective in getting rid of the contaminated samples, reduces the computational complexity significantly, and promotes the target detection performance compared with the method of GIP (generalized inner product).
\end{abstract}

\section{Introduction}

The homogeneous clutter environment is the prerequisite of STAP to suppress ground/sea clutter and detect targets effectively [1-3]. In terms of the classical STAP $[4,5]$, obtaining the CCM (clutter covariance matrix) is important. CCM is estimated by the training samples that have the IID (independent and identically distributed) feature with the CUT (cell under test). In general, the number of IID training samples needs to be twice the DOF (degrees of freedom) of the system in order to get the approximate optimal performance [6]. This can be ensured in the homogeneous clutter environment. However, in the actual condition, the heterogeneity of clutter affects the IID relationship between the training samples and the CUT. Furthermore, the great reduction in the number of satisfactory samples leads to inaccurate CCM estimation. Finally, the target detection performance of STAP is obviously deteriorated [7-11].
Training samples containing target-like signals, namely, the samples contaminated by the target-like signals, is one of the major factors that cause clutter heterogeneity $[12,13]$. Before calculating the CCM, the interference detection of each sample is required, because the existing contaminated samples have a bad influence on the estimated accuracy of CCM [14]. Hence, it is important to find a more effective method to solve the interference detection problem. At present, GIP (generalized inner product) is a common method which builds the test statistics by calculating the inversion of CCM to pick out the contaminated samples [15]. GIP is available when fewer target-like signals are contained or the jamming intensity of the contained ones is smaller. Nevertheless, in the condition of target-like signals with big jamming intensity, for example, when JNR (jamming noise ratio) is $20 \mathrm{~dB}$ greater than SNR (signal-to-noise ratio), there is no possibility of picking out the contaminated samples due to the obvious inaccuracy in CCM estimation and the 
dramatic fluctuation in test statistics. Meanwhile, the CCM estimation and its inversion are required in the processing of GIP, which lead to the heavy computational complexity of STAP and go against efficient target detection. In addition, SR (sparse recovery) is another solution. Recently, considering the sparse physical property of clutter in space-time plane, SR is applied in STAP $[6,16]$. As for SR-STAP, the spacetime spectrum of CUT is directly estimated to calculate the CCM, which avoids the contamination problem of training samples. However, high computational complexity has arisen from sparse grid partition, and clutter suppression performance deterioration caused by big CCM estimation error has appeared when the isolated interference signals exist in the CUT.

Based on the above analysis, TT (time-time) transform is considered to solve the interference detection problem of the training samples in this paper [17-19]. TT transform was proposed by Pinnegar and Mansinha $[20,21]$ as a new transform in 2003, which came from the inverse Fourier form of the time-frequency analysis $S$ transform [22-25]. Onedimensional time series is expressed as a two-dimensional time-time series by TT transform, which is good for observing the local features of the signal [26]. An important feature of TT transform is that the main energy of the signal can be focalized in the main diagonal position [27]. In this letter, the signal energy in the main diagonal position is only extracted to realize the rejection of the contaminated samples and the reduction of the computational cost. The method is called simplified TT transform. When the training samples are contaminated by some target-like signals with bigger jamming intensity, the energy of these samples varies greatly between the unpolluted stage and the polluted stage. Therefore, a kind of interference detection method based on simplified TT transform is put forward from the viewpoint of time-domain energy in this letter. Firstly, the data on each training sample is converted into one-dimensional discrete slow time series, respectively. Then, the transform-spectrum energy of each series is extracted to separate the contaminated samples and the clean samples in the simplified TT transform domain. Lastly, the polluted training samples are rejected. Through the above treatment, the target detection performance on STAP is improved and the total computational cost is reduced in the heterogeneous clutter environment.

\section{Signal Model of STAP}

Assuming the side-looking antenna array is $M \times N$ in the airborne radar, this array is shown as the $N$ uniform linear arrays via the column equivalent synthesis. $K$ pulses are contained in a CPI (coherent processing interval) and the number of the observed range cells is $Q$. The data sampling process about STAP can be described by the element-pulserange domain, and then data collection is composed of $N \times$ $K \times Q$ sampling points. Each range cell is a matrix of $N \times K$. If the matrix is converted into a vector of $N K \times 1$ which corresponds with the slow time (pulse domain), a slow time sequence of each range cell is obtained. Its specific form is denoted in Figure 1.

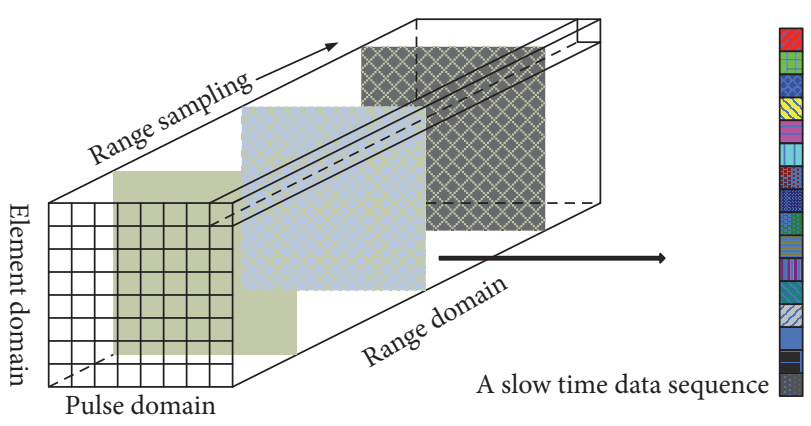

FIGURE 1: Data sampling process of STAP.

As for Figure 1, the data matrix about the $q$ th $(1,2, \ldots, Q)$ range cell is expressed as

$$
\mathbf{X}_{q}=\left(\begin{array}{cccc}
x_{q 11} & x_{q 21} & \cdots & x_{q N 1} \\
x_{q 12} & x_{q 22} & \cdots & x_{q N 2} \\
\vdots & \vdots & \ddots & \vdots \\
x_{q 1 K} & x_{q 2 K} & \cdots & x_{q N K}
\end{array}\right)
$$

By vector conversation, (1) has the following form:

$$
\operatorname{vec}\left(\mathbf{X}_{q}\right)=\left(x_{q 11}, \ldots, x_{q N 1}, x_{q 12}, \ldots, x_{q N 2}, \ldots, x_{q N K}\right) \text {. }
$$

$\operatorname{vec}\left(\mathbf{X}_{q}\right)$ is the sampling data of the $q$ th $(1,2, \ldots, Q)$ range cell in the different elements and different pulses. Considering the sparse physical property of clutter in spacetime plane, $\operatorname{vec}\left(\mathbf{X}_{q}\right)$ is discretized by the sampling interval of different elements in the same pulse. Supposing $\mathbf{g}_{N \times K}$ is a discrete time sequence, $\mathbf{g}_{N \times K}$ is one-to-one mapping with $\operatorname{vec}\left(\mathbf{X}_{q}\right)$. Then, the relevant discrete slow time data sequence of $\operatorname{vec}\left(\mathbf{X}_{q}\right)$ can be expressed as

$$
f\left(\mathbf{g}, \operatorname{vec}\left(\mathbf{X}_{q}\right)\right)=\left(x\left(g_{1}\right), x\left(g_{2}\right), \ldots, x\left(g_{N \times K}\right)\right) .
$$

If $f\left(\mathbf{g}, \operatorname{vec}\left(\mathbf{X}_{q}\right)\right)$ is replaced with $\mathbf{W}_{q}$ and the CUT is indicated by $q_{0}$, the data in the CUT is denoted as

$$
\mathbf{W}_{q_{0}}=\sum_{i=1}^{N_{c}} \rho_{c i}^{q_{0}} \mathbf{s}_{c}^{q_{0}}\left(\bar{w}_{t i}, \bar{w}_{s i}\right)+\rho_{t}^{q_{0}} \mathbf{s}_{t}^{q_{0}}\left(\bar{\chi}_{t}, \bar{\chi}_{s}\right)+\mathbf{n}_{q_{0}},
$$

where $N_{c}$ is the number of clutter patches; $\rho_{c i}^{q_{0}}$ and $\mathbf{s}_{c}^{q_{0}}\left(\bar{w}_{t i}, \bar{w}_{s i}\right)$ are the reflected coefficient of the $i$ th clutter patch and its space-time steering vector, respectively; $\bar{w}_{t i}$ and $\bar{w}_{s i}$ show the normalized Doppler frequency of the $i$ th clutter patch and its normalized spatial frequency, respectively; $\rho_{t}^{q_{0}}$ and $s_{t}^{q_{0}}\left(\bar{\chi}_{t}, \bar{\chi}_{s}\right)$ are the reflected coefficient of the target in the CUT and its space-time steering vector, respectively; $\bar{\chi}_{t}$ and $\bar{\chi}_{s}$ show the normalized Doppler frequency of the target in the CUT and its normalized spatial frequency, respectively; $\mathbf{n}_{q_{0}}$ is the noise.

The data about each training cell is defined as

$$
\begin{aligned}
\mathbf{W}_{q}= & \sum_{i=1}^{N_{c}} \rho_{c i}^{q} \mathbf{s}_{c}^{q}\left(\bar{w}_{t i}, \bar{w}_{s i}\right)+\left(\alpha_{q}-1\right) \sigma_{g}^{q} \zeta_{g}^{q}\left(\bar{\chi}_{g t}, \bar{\chi}_{g s}\right) \\
& +\mathbf{n}_{q},
\end{aligned}
$$


where the value range of $q$ is $0<q \leq Q$, which is unequal to $q_{0}$; the polluted situation about each sample is reflected by $\alpha_{q}$; when $\alpha_{q}$ is equal to 1 , the samples are unpolluted; when $\alpha_{q}$ is bigger than 1, the samples are polluted; $\sigma_{g}^{q}$ and $\zeta_{g}^{q}\left(\bar{\chi}_{g t}, \bar{\chi}_{g s}\right)$ denote the reflected coefficient of the target-like signal and its space-time steering vector, respectively; $\bar{\chi}_{g t}$ and $\bar{\chi}_{g s}$ show the normalized Doppler frequency of the target-like signal and its normalized spatial frequency, respectively; $\mathbf{n}_{q}$ is the noise.

Combined with the above signal model, two kinds of interference detection methods are discussed. In the following analysis and comparison, the interference detection methods of STAP using GIP and simplified TT transform are simply called GIP-STAP and simplified TT-STAP, respectively.

\section{Interference Detection Method of GIP-STAP}

Detecting the contaminated training samples $\mathbf{W}_{q}$ by GIPSTAP, the test covariance matrix $\widehat{\mathbf{R}}_{q}$ is firstly constructed to realize the prewhitening processing about $\mathbf{W}_{q}$ and get $\widehat{\mathbf{W}}_{q}$. Then, the test statistics of each training sample are calculated in order to pick out the heterogeneous samples caused by the target-like signals. The test statistics about GIP-STAP are defined as

$$
\gamma_{q}=\widehat{\mathbf{W}}_{q}^{\mathrm{H}} \widehat{\mathbf{W}}_{q}=\left(\mathbf{W}_{q}^{\mathrm{H}} \widehat{\mathbf{R}}_{q}^{-1 / 2}\right)\left(\widehat{\mathbf{R}}_{q}^{-1 / 2} \mathbf{W}_{q}\right)=\mathbf{W}_{q}^{\mathrm{H}} \widehat{\mathbf{R}}_{q}^{-1} \mathbf{W}_{q} .
$$

According to (6), before the test statistics about GIPSTAP are obtained, calculating the prior covariance matrix $\widehat{\mathbf{R}}_{q}$ is firstly required. The estimation error of $\widehat{\mathbf{R}}_{q}$ directly affects the performance of detecting the polluted samples. If there are many training samples contaminated by the targetlike signals with big jamming intensity, the error of the test statistics increases seriously, which leads to the failure of rejecting the polluted samples. Meanwhile, the computational complexity is obviously sharpened because of estimating the CCM and calculating its inversion.

In terms of the above problem, the following part adopts the simplified TT transform to get rid of the polluted samples from the viewpoint of energy-variant difference between the unpolluted stage and the polluted stage.

\section{Interference Detection Method of Simplified TT-STAP}

4.1. Simplified TT Transform. Before the definition of simplified TT transform is given, TT transform is firstly introduced. Concerning the continuous signal $\mu(t)$, its TT transform is expressed as

$$
\begin{aligned}
\operatorname{TT} & (t, \tau) \\
& =\int_{-\infty}^{+\infty} \int_{-\infty}^{+\infty} \mu(\vartheta) \frac{|f|}{\varepsilon \sqrt{2 \pi}} e^{-[f(t-\vartheta) / \sqrt{2} \varepsilon]^{2}} e^{i 2 \pi f(\vartheta-\tau)} d \vartheta d f \\
& =\int_{-\infty}^{+\infty} \mu(\vartheta) d \vartheta \int_{-\infty}^{+\infty} \frac{|f|}{\varepsilon \sqrt{2 \pi}} e^{-[f(\vartheta-t) / \sqrt{2} \varepsilon]^{2}} e^{i 2 \pi f(\vartheta-\tau)} d f,
\end{aligned}
$$

where $t$ and $\tau$ denote time; $f$ indicates frequency; $(|f| / \varepsilon \sqrt{2 \pi}) e^{-[f(t-9) / \sqrt{2} \varepsilon]^{2}}$ means the Gaussian window function; $\varepsilon$ is the regulatory factor of window scale.

Assuming $\eta$ and $\nu$ are equal to $\vartheta-t$ and $\vartheta-\tau$, respectively,

(7) is arranged as

$$
\begin{aligned}
\operatorname{TT} & (t, \tau) \\
& =\int_{-\infty}^{+\infty} \mu(\vartheta) d \vartheta \int_{-\infty}^{+\infty} \frac{|f|}{\varepsilon \sqrt{2 \pi}} e^{-[f \eta / \sqrt{2} \varepsilon]^{2}} e^{i 2 \pi f v} d f,
\end{aligned}
$$

where

$$
\begin{aligned}
& \int_{-\infty}^{+\infty} \frac{|f|}{\varepsilon \sqrt{2 \pi}} e^{-[f \eta / \sqrt{2} \varepsilon]^{2}} e^{i 2 \pi f v} d f \\
& = \begin{cases}\frac{\varepsilon}{\eta^{2}} \sqrt{\frac{2}{\pi}}-\frac{2 \pi \varepsilon^{2} v}{\eta^{3}} e^{-2 \pi^{2} \nu^{2} \varepsilon^{2} / \eta^{2}} \text { erfi }\left(\frac{\sqrt{2} \varepsilon \pi v}{\eta}\right) & \eta \neq 0 \\
-\sqrt{\frac{2}{\varepsilon^{2} \pi v^{4}}} & \eta=0,\end{cases}
\end{aligned}
$$

where $\operatorname{erfi}(\bullet)$ is the imaginary error function.

Combining (9) with (8), TT transform is

$$
\operatorname{TT}(t, \tau)= \begin{cases}\int_{-\infty}^{+\infty} \mu(\vartheta)\left[\frac{\varepsilon}{(\vartheta-t)^{2}} \sqrt{\frac{2}{\pi}}-\frac{2 \pi \varepsilon^{2}(\vartheta-\tau)}{(\vartheta-t)^{3}} e^{-2 \pi^{2}(\vartheta-\tau)^{2} \varepsilon^{2} /(\vartheta-t)^{2}} \operatorname{erfi}\left(\frac{\sqrt{2} \varepsilon \pi(\vartheta-\tau)}{\vartheta-t}\right)\right] d \vartheta, & \vartheta \neq t \\ \int_{-\infty}^{+\infty}-\mu(\vartheta) \sqrt{\frac{2}{\varepsilon^{2} \pi(\vartheta-\tau)^{4}}} d \vartheta & \vartheta=t .\end{cases}
$$

We simplify (10) to

$$
\operatorname{TT}(t, \tau)= \begin{cases}\int_{-\infty}^{+\infty} \frac{\mu(\vartheta)}{(\vartheta-t)^{2}}\left[\varepsilon \sqrt{\frac{2}{\pi}}-\frac{2 \pi \varepsilon^{2}(\vartheta-\tau)}{\vartheta-t} e^{-2 \pi^{2}(\vartheta-\tau)^{2} \varepsilon^{2} /(\vartheta-t)^{2}} \operatorname{erfi}\left(\frac{\sqrt{2} \varepsilon \pi(\vartheta-\tau)}{\vartheta-t}\right)\right] d \vartheta, & \vartheta \neq t \\ \int_{-\infty}^{+\infty}-\frac{\mu(\vartheta)}{\varepsilon(\vartheta-\tau)^{2}} \sqrt{\frac{2}{\pi}} d \vartheta & \vartheta=t .\end{cases}
$$


TABLE 1: Analysis on computational complexity.

\begin{tabular}{lccc}
\hline Methods & CCM estimation & Matrix inversion & Simplified TT transform \\
\hline GIP-STAP & $O\left(Q(N K)^{2}\right)$ & $O\left((N K)^{3}\right)$ & - \\
Simplified TT-STAP & - & - & $O(N K Q)$ \\
\hline
\end{tabular}

Hence,

$$
\begin{aligned}
\delta & =\frac{\vartheta-\tau}{\vartheta-t}, \\
\xi(\delta) & =\varepsilon \sqrt{\frac{2}{\pi}}-2 \pi \varepsilon^{2} \delta e^{-2 \pi^{2} \varepsilon^{2} \delta^{2}} \operatorname{erfi}(\sqrt{2} \varepsilon \pi \delta) .
\end{aligned}
$$

Furthermore, (11) is denoted as

$$
\mathrm{TT}(t, \tau)= \begin{cases}\int_{-\infty}^{+\infty} \frac{\mu(\vartheta)}{(\vartheta-t)^{2}} \xi(\delta) d \vartheta, & \vartheta \neq t \\ \int_{-\infty}^{+\infty}-\frac{\mu(\vartheta)}{\varepsilon(\vartheta-\tau)^{2}} \sqrt{\frac{2}{\pi}} d \vartheta, & \vartheta=t\end{cases}
$$

In a physical way, (13) is explained in such a way that the function $\xi(\delta)$ is rapidly convergent to 0 because of $\delta>1$. Meanwhile, considering that time is nonnegative, the main energy about $\xi(\delta)$ is contained in the region of $|\delta| \leq 1(0 \leq$ $\tau \leq t$ ). For $\tau=t$, the main energy has been maximally reflected, which indicates that the main energy about the signal $\mu(t)$ is presented in the main diagonal position of TT transform domain. Summing up the above analyses, extracting the diagonal feature of TT transform spectrum about $\mu(t)$ can obtain the main energy of $\mu(t)$ in the time domain.

In terms of $\tau=t, \mathrm{TT}(t, \tau)$ transform is changed into a simplified form that is called simplified TT transform. Combined with (13), the simplified TT transform is defined as

$$
\mathrm{TT}(t, t)=\int_{-\infty}^{+\infty} \frac{\mu(\vartheta)}{(\vartheta-t)^{2}} \xi(1) d \vartheta, \quad \vartheta \neq t
$$

4.2. Interference Detection Method. Since the discrete data is often adopted in the processing of STAP, converting simplified TT transform into the discrete form is necessary. Discretization about (14) is expressed as

$$
\mathrm{TT}(k T, k T)=\sum_{j=0}^{P-1} \xi(1) \frac{\mu(j T)}{(j T-k T)^{2}}, \quad j T \neq k T,
$$

where $T$ is the time sampling interval; $P$ is the number of sampling points; $k=0,1, \ldots, P-1$.

Combining (4) with (5), the dimension of a range cell is $N K \times 1$. So, the number of sampling points is $N K$. Discrete simplified TT transform of $\mathbf{W}_{q}$ has the following form:

$$
\mathrm{TT}_{\text {stap }}(k T, k T)=\sum_{j=0}^{N K-1} \xi(1) \frac{\mathbf{W}_{q}(j T)}{(j T-k T)^{2}}, \quad j T \neq k T,
$$

where $0<q \leq Q$.
Based on the physical property analysis about TT transform in Section 4.1, the energy of the signal in the time domain is mainly focused in the diagonal position of the TT transform, which is regarded as the simplified TT transform spectrum of the signal. When the clutter presents the heterogeneity caused by the polluted training samples, the energy about training samples produces a valid change. Meanwhile, the energy difference between the polluted samples and the unpolluted samples increases. By means of this feature, extracting the energy of training samples using (16) can realize the effective rejection of the training samples that contain the target-like signals.

\section{Computational Complexity Analyses}

In STAP, the computation load is one of the most important standards to judge the performance of interference detection methods. Smaller computation load means better application in actual environments. Estimating the CCM and getting its inversion are the two main steps of aggravating the computational complexity in STAP. Before comparing the performance of GIP-STAP with that of simplified TT-STAP, the computational load analysis is firstly denoted in Table 1.

The computational load about simplified TT-STAP reduces rapidly because CCM estimation and its inversion are not required in the jamming detection. Using simplified TT-STAP, the total computational load descends from $O\left(Q(N K)^{2}\right)+O\left((N K)^{3}\right)$ to $O(N K Q)$, which promotes the processing of STAP obviously.

\section{Simulations}

In order to compare GIP-STAP with simplified TT-STAP in terms of detection performance, several simulation results have been presented in this part. The main parameters are set in Table 2.

Based on the parameters in Table 2, the simulation results are presented as follows.

6.1. Comparison on the Test Statistics. Concerning detection about the contaminated samples, the test statistics of each training sample are plotted in Figures 2 and 3.

Considering the 150th sample as CUT and its both sides as a protecting unit, these cells are ignored in the experiments. Thus, the test statistics results about 237 training samples are presented in the two figures. Comparing Figure 2 with Figure 3, when four target-like signals with big jamming intensity are contained in the training samples, the precision of estimating CCM by GIP-STAP decreases evidently, which leads to the large fluctuation about the statistics value and detecting the polluted samples invalidly. As for simplified 
TABle 2: Parameter setting.

\begin{tabular}{lc}
\hline Parameter & Value \\
\hline Radar array & $10 \times 10$ \\
Velocity of plane & $140 \mathrm{~m} / \mathrm{s}$ \\
Height of plane & $6000 \mathrm{~m}$ \\
Number of pulses & 12 \\
PRF (pulse repetition frequency) & $2434.8 \mathrm{~Hz}$ \\
Element distance & $0.115 \mathrm{~m}$ \\
Wavelength & $0.23 \mathrm{~m}$ \\
CNR & $60 \mathrm{~dB}$ \\
Number of training samples & 240 \\
Position of CUT & 150 \\
SNR & $10 \mathrm{~dB}$ \\
JNR & $30 \mathrm{~dB}$ \\
Position of the contaminated samples & $30,90,210,230$ \\
Number of Monte Carlo simulations & 1000 \\
\hline
\end{tabular}

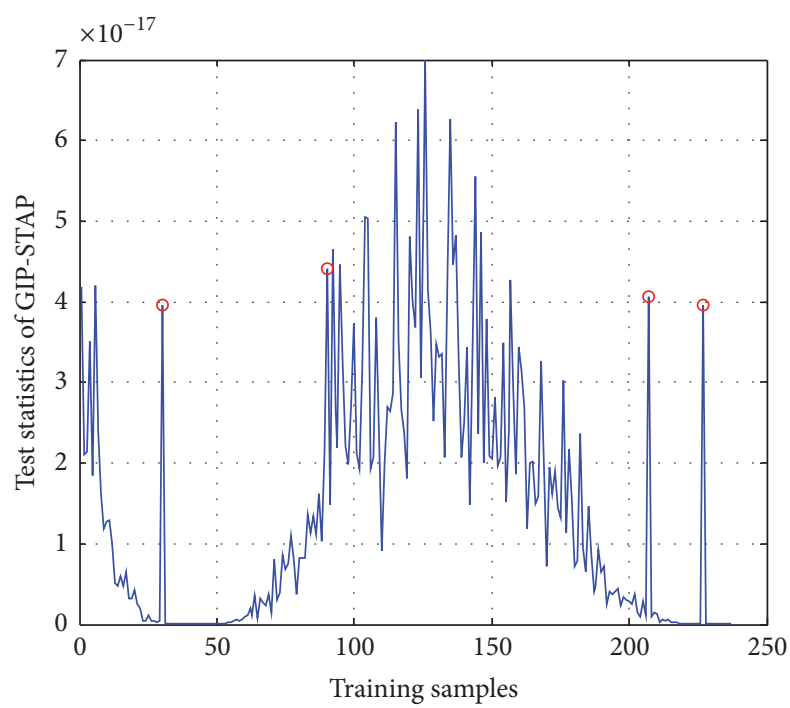

FIGURE 2: Test statistics of GIP-STAP.

TT-STAP, it avoids CCM estimation and the four contaminated samples can be clearly seen, which is good for realizing the polluted samples rejection.

For ease of the physical explanation, the energy variance of each polluted sample between the polluted stage and the unpolluted stage is analyzed as follows.

6.2. Simplified TT Transform Spectrum. Simplified TT transform spectrums of the four training samples are, respectively, denoted in Figures 4, 5, 6, and 7.

According to Figures 4-7, simplified TT transform spectrums change obviously between the unpolluted stage and the polluted stage. In terms of the same cell, the color of the simplified TT transform spectrum deepens from the unpolluted stage to the polluted stage, which has a direct relation with the energy distribution of the sample. Moreover, the power of the simplified TT transform spectrum in each sample is increased by at least an order of magnitude.

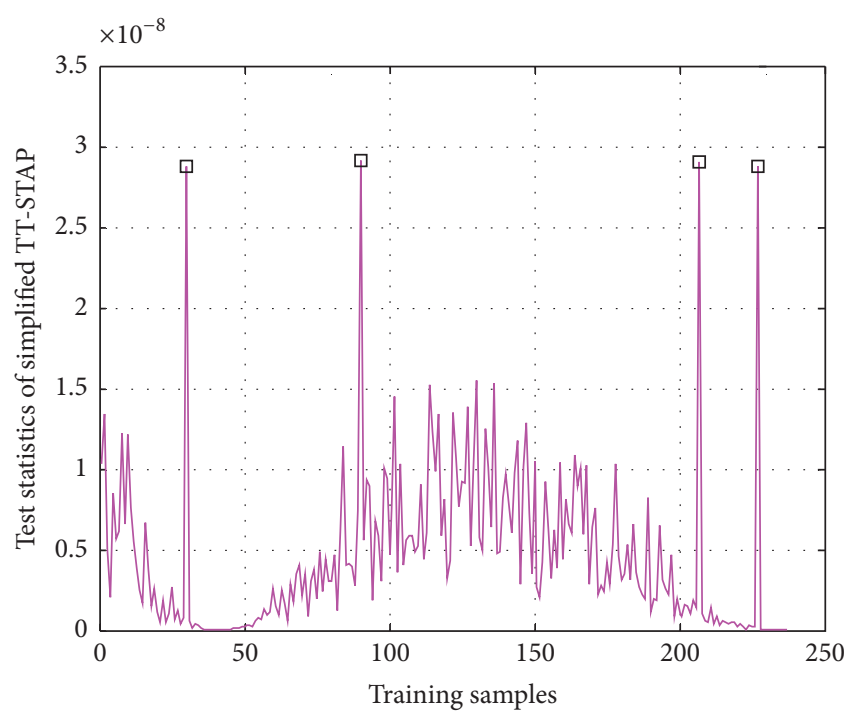

FIgUre 3: Test statistics of simplified TT-STAP.

Hence, it is ensured that simplified TT-STAP can detect the contaminated samples effectively from the viewpoint of energy.

6.3. Comparison on the Output Power. Combined with the same simulation data, the output power of STAP is simulated between the unpolluted stage and the polluted stage in this part. Meanwhile, comparison on the output power using GIP-STAP and simplified TT-STAP is made. The results are denoted in Figures 8 and 9.

Analyzing Figures 8 and 9, there are two points we can get: (1) in terms of Figure 8, when the contaminated training samples exist, estimating the CCM is affected and the error of the adaptive weight increases. Furthermore, the output power deteriorates seriously after STAP and the target in the CUT cannot be obtained; (2) as for Figure 9, the prior covariance matrix estimation is required about GIP-STAP. If there are some target-like signals with big interference intensity in the training samples, the error of estimating the CCM exists and the polluted samples are not rejected effectively. So, the output power is not improved by GIP-STAP and the real target is detected invalidly. Aiming at simplified TT-STAP, it analyzes the training samples from the viewpoint of energy. The matrix error has no influence on jamming detection, and then the four polluted samples are eliminated and the real target is obtained.

6.4. Comparison on the Detection Performance. The final purpose of STAP is to detect the target in a strong clutter environment. In this part, simulations about IF (improved factor), the gap between primary peak of the output power and its secondary peak, the output SCR (signal clutter ratio), and PD (probability of detection) are made. The results are presented in Figures 10, 11, 12, and 13.

As for GIP-STAP in Figure 10, the deep null is formed not only in the main clutter region, but also in the Doppler frequency of the real target because of the contaminated 


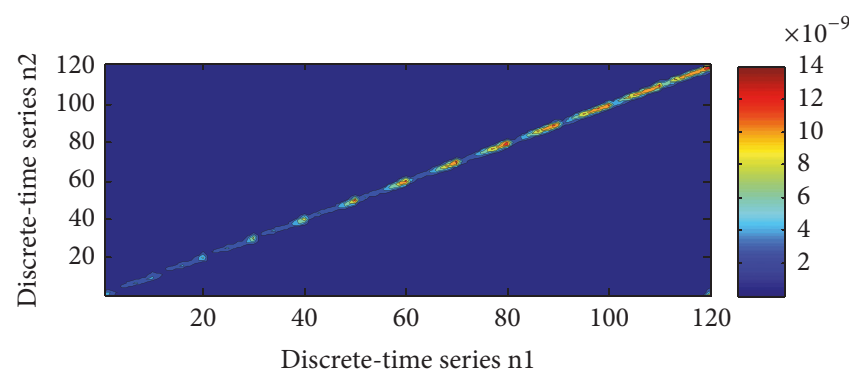

(a) Uncontaminated simplified TT transform spectrum

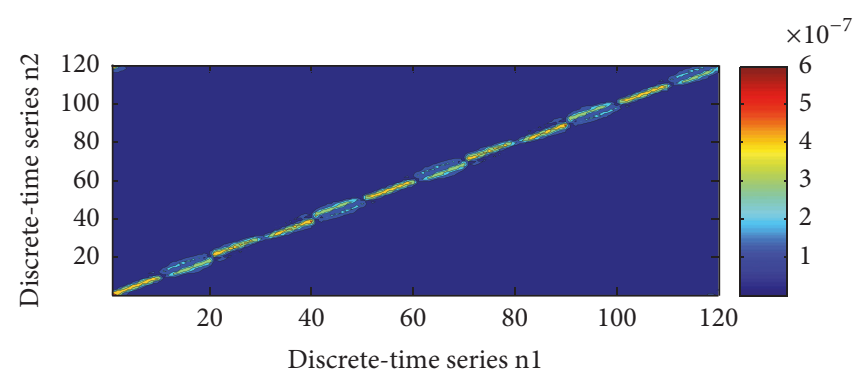

(b) Contaminated simplified TT transform spectrum

FIGURE 4: Energy distribution of the 30th range cell.

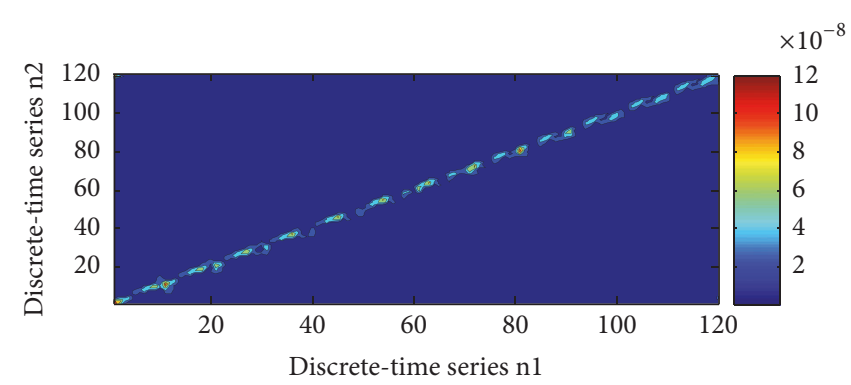

(a) Uncontaminated simplified TT transform spectrum

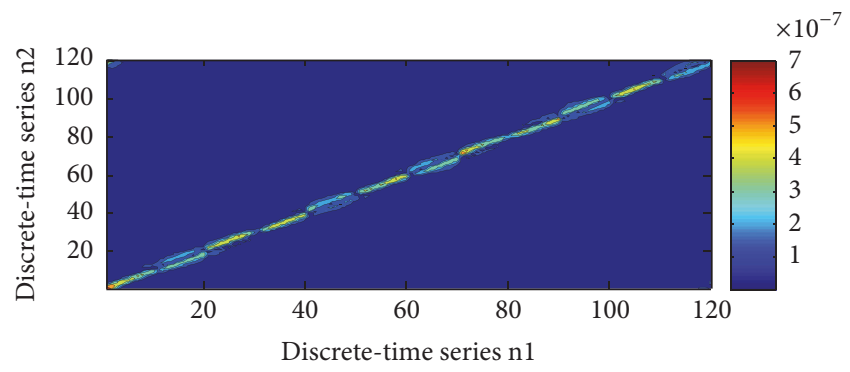

(b) Contaminated simplified TT transform spectrum

FIGURE 5: Energy distribution of the 90th range cell.

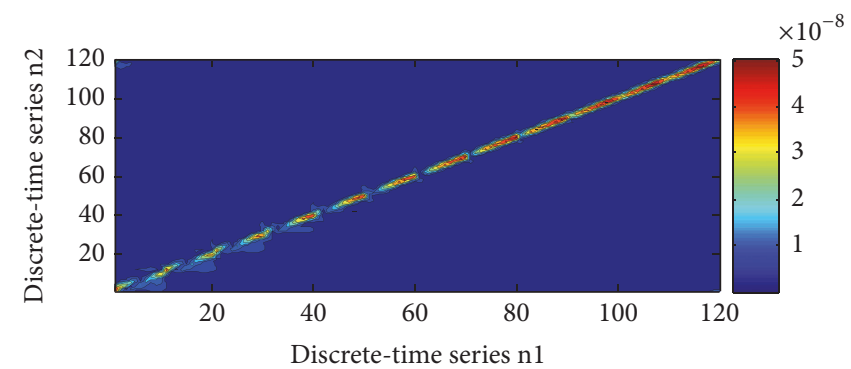

(a) Uncontaminated simplified TT transform spectrum

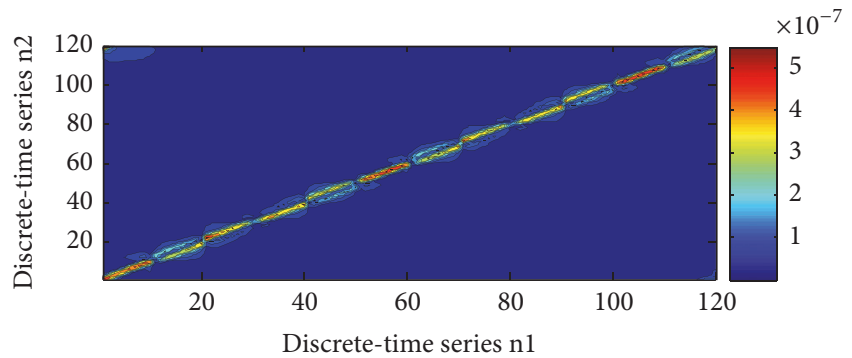

(b) Contaminated simplified TT transform spectrum

FIGURE 6: Energy distribution of the 210th range cell.

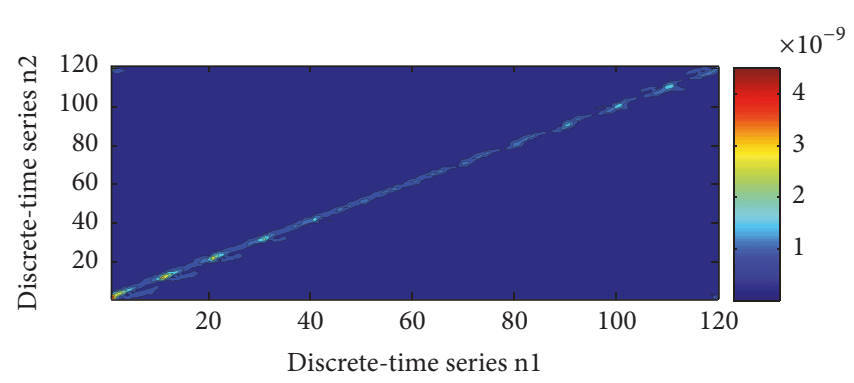

(a) Uncontaminated simplified TT transform spectrum

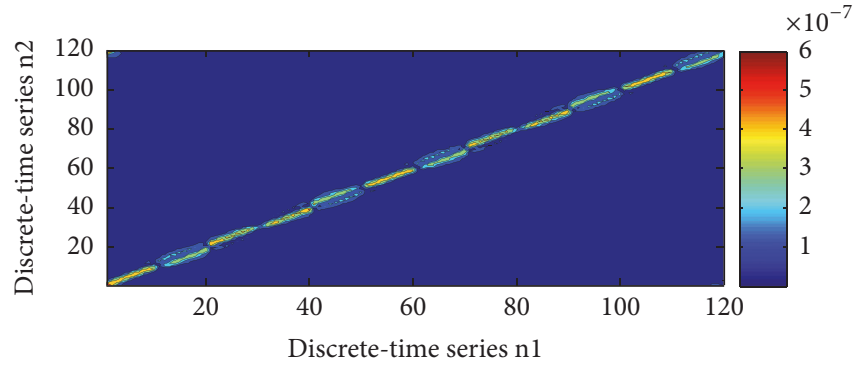

(b) Contaminated simplified TT transform spectrum

FIGURE 7: Energy distribution of the 230th range cell.

samples rejected invalidly. And then the target detection performance decreases obviously. However, the four contaminated samples are all rejected by simplified TT-STAP. Estimating the CCM is not affected and the deep null is only formed in the main clutter region. Furthermore, the clutter suppression performance is obviously improved.

In terms of Figure 11, the gap between the primary peak and secondary peak reflects the target detection performance 


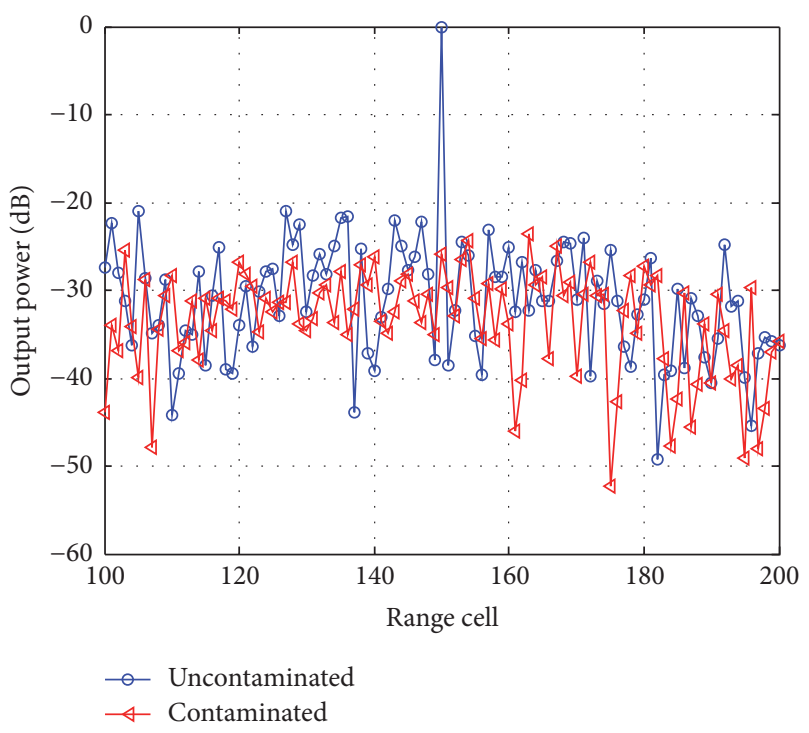

FIGURE 8: Output power changing with range cell before jamming detection.

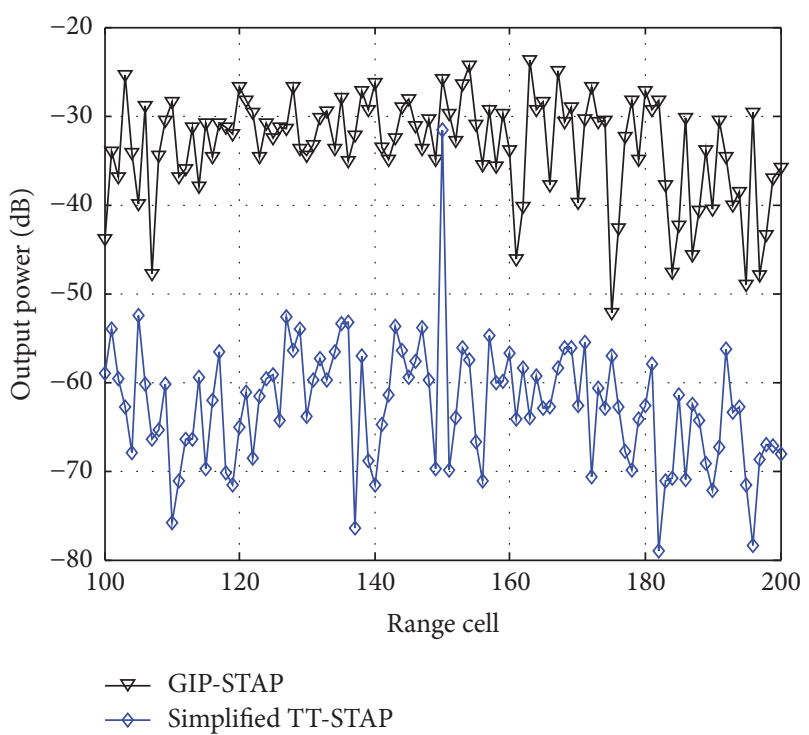

FIGURE 9: Comparison on the output power after jamming detection.

after interference detection. The target is more easily detected if the gap is bigger. With the increase of INSCR, the gap is promoted, which means the target detection performance is improved gradually. Meanwhile, in the same INSCR, simplified TT-STAP is superior to GIP-STAP. When INSCR is smaller, the performances of the two methods are equivalent because the signal is weak. As the signal becomes stronger, the performance of GIP-STAP is improved but is still inferior to the simplified TT-STAP.

In Figure 12, OUTSCR and INSCR of CUT have a positive correlation. In the same INSCR, the OUTSCR improvement of simplified TT-STAP is more obvious than that of GIPSTAP. Since the target-like signals are rejected ineffectively

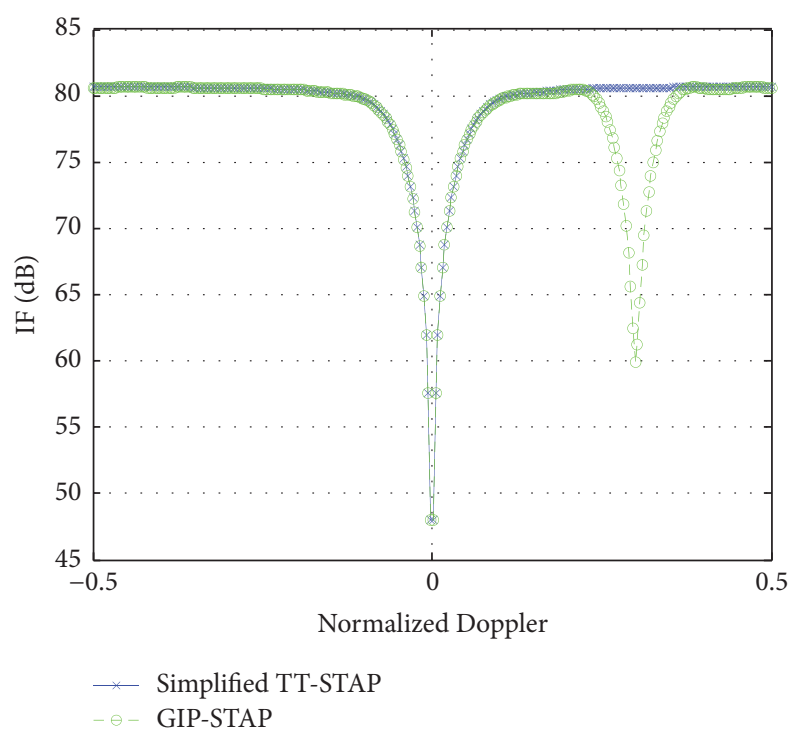

FIGURE 10: Relation between IF and normalized Doppler frequency.

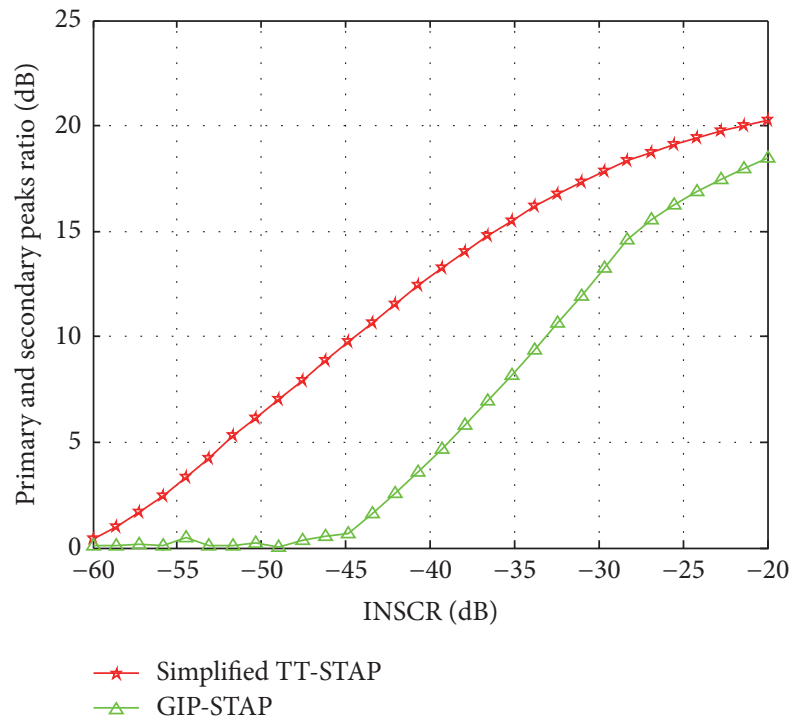

FIGURE 11: Difference between primary peak and secondary peak of the output power.

using GIP-STAP, the error of the adaptive weight increases, which leads to a disappointing OUTSCR improvement.

Based on the output SCR, the relation between PD and INSCR is given in Figure 13 using CFAR. In the same false alarm probability, $\mathrm{PD}$ is promoted gradually with increases in INSCR. Since estimating the CCM is inaccurate by GIPSTAP, PD of GIP-STAP is more inferior than that of simplified TT-STAP with the same INSCR.

\section{Conclusions}

The inhomogeneous clutter environment appears when training samples are contaminated by some target-like signals with big jamming intensity. In order to eliminate the bad influence 


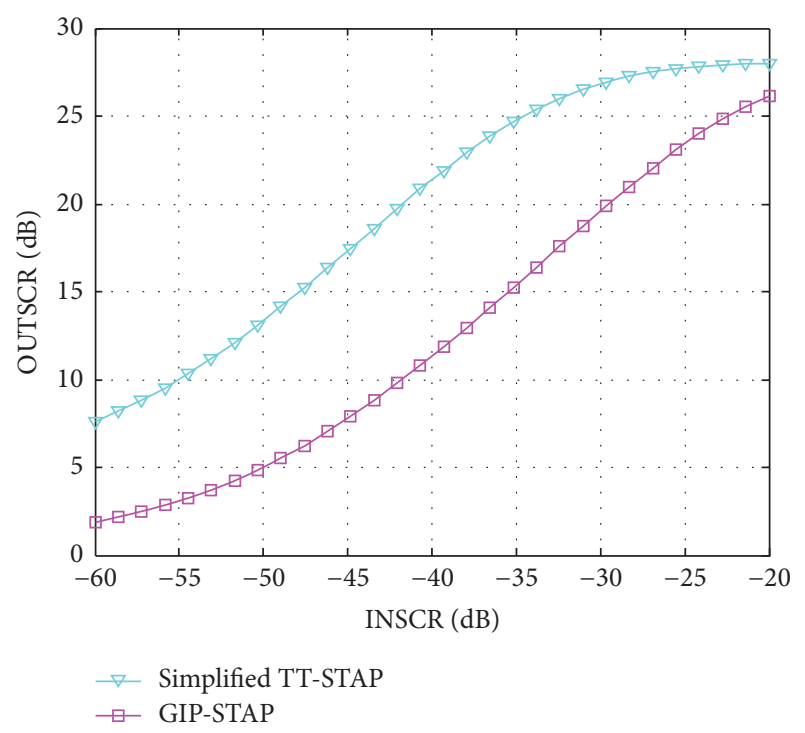

FIGURE 12: Relation between INSCR and OUTSCR.

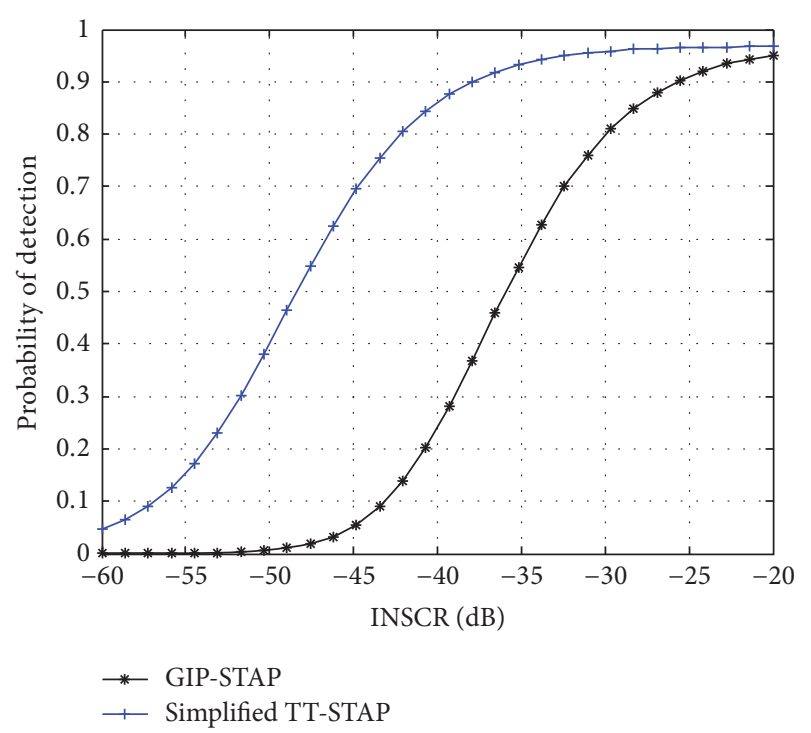

FIgURE 13: Relation between INSCR and PD.

of such environment, an interference detection method based on simplified TT transform is proposed from the viewpoint of energy in time domain. Combined with the sparse physical property of clutter in the space-time plane, the training samples are firstly converted into discrete slow time sequences. Then, the reason of getting rid of the polluted samples invalidly is analyzed. Thirdly, the formula about simplified TT transform is derived based on TT transform and the physical explanation of rejecting the polluted samples by simplified TT-STAP is given. Fourthly, the computational complexity of each method is discussed. At last, the performances on picking out the polluted samples and detecting the real target are verified. Compared with GIP-STAP, the proposed method is more effective, which avoids the influence of the polluted samples on the adaptive weight and reduces the computational load of STAP. Furthermore, simplified TTSTAP has a better practical value and theoretical research significance.

\section{Conflicts of Interest}

The authors declare that there are no conflicts of interest regarding the publication of this paper.

\section{Acknowledgments}

This work was supported in part by the National Natural Science Foundation of China under Grant 61501501.

\section{References}

[1] Z. Wang, Y. Wang, K. Duan, and W. Xie, "Subspace-augmented clutter suppression technique for STAP radar," IEEE Geoscience and Remote Sensing Letters, vol. 13, no. 3, pp. 462-466, 2016.

[2] W. Wang, Z. Chen, X. Li, and B. Wang, "Space time adaptive processing algorithm for multiple-input-multiple-output radar based on Nyström method," IET Radar, Sonar \& Navigation, vol. 10, no. 3, pp. 459-467, 2016.

[3] Z. Gao, H. Tao, S. Zhu, and J. Zhao, "L1-regularised joint iterative optimisation space-time adaptive processing algorithm," IET Radar, Sonar \& Navigation, vol. 10, no. 3, pp. 435-441, 2016.

[4] L. E. Brennan and L. S. Reed, "Theory of adaptive radar," IEEE Transactions on Aerospace and Electronic Systems, vol. 9, no. 2, pp. 237-252, 1973.

[5] T. Wang, Y. Zhao, T. Lai, and J. Wang, "Performance analysis of polarization-space-time adaptive processing for airborne polarization array multiple-input multiple-output radar," Acta Physica Sinica, vol. 66, no. 4, pp. 048401(1)-048401(9), 2017.

[6] Z. Ma, X. Wang, Y. Liu, and H. Meng, "An overview on sparse recovery-based STAP," Journal of Radars, vol. 3, no. 2, pp. 217228, 2014.

[7] Y. Tong, T. Wang, and J. Wu, "Improving EFA-STAP performance using persymmetric covariance matrix estimation," IEEE Transactions on Aerospace and Electronic Systems, vol. 51, no. 2, pp. 924-936, 2015.

[8] Y. Wu, T. Wang, J. Wu, and J. Duan, "Robust training samples selection algorithm based on spectral similarity for spacetime adaptive processing in heterogeneous interference environments," IET Radar, Sonar \& Navigation, vol. 9, no. 7, pp. 778782,2015

[9] Y. Wu, T. Wang, J. Wu, and J. Duan, “Training sample selection for space-time adaptive processing in heterogeneous environments," IEEE Geoscience and Remote Sensing Letters, vol. 12, no. 4, pp. 691-695, 2015.

[10] J. H. Bang, W. L. Melvin, and A. D. Lanterman, "Model-based clutter cancellation based on enhanced knowledge-aided parametric covariance estimation," IEEE Transactions on Aerospace and Electronic Systems, vol. 51, no. 1, pp. 154-166, 2015.

[11] C. Hao, S. Gazor, D. Orlando, G. Foglia, and J. Yang, "Parametric space-time detection and range estimation of a small target," IET Radar, Sonar \& Navigation, vol. 9, no. 2, pp. 221-231, 2015.

[12] L. B. Fertig, "Analytical expressions for space-time adaptive processing (STAP) performance," IEEE Transactions on Aerospace and Electronic Systems, vol. 51, no. 1, pp. 442-453, 2015. 
[13] W. Zhang, Z. He, J. Li, and C. Li, "Beamspace reduceddimension space-time adaptive processing for multiple-input multiple-output radar based on maximum cross-correlation energy," IET Radar, Sonar \& Navigation, vol. 9, no. 7, pp. 772$777,2015$.

[14] K. Duan, W. Xie, Y. Wang, W. Liu, and F. Gao, "A deterministic auto-regressive STAP approach for nonhomogenerous clutter suppression," Multidimensional Systems and Signal Processing. An International Journal, vol. 27, no. 1, pp. 105-119, 2016.

[15] T. Wang and Y. Zhao, "Knowledge-aided non-homogeneous samples detection method for airborne MIMO radar STAP," $X i$ Tong Gong Cheng Yu Dian Zi Ji Shu, vol. 37, no. 10, pp. 22602265, 2015.

[16] Z. Wang, K. Duan, W. Xie, and Y. Wang, "A joint sparse recovery STAP method based on SA-MUSIC," Tien Tzu Hsueh Pao/Acta Electronica Sinica, vol. 43, no. 5, pp. 846-853, 2015.

[17] S. Suja and J. Jerome, "Pattern recognition of power signal disturbances using S Transform and TT Transform," International Journal of Electrical Power \& Energy Systems, vol. 32, no. 1, pp. 37-53, 2010.

[18] J. Ma and Q. Li, "Surface Wave Suppression with Joint S Transform and TT Transform," Procedia Earth and Planetary Science, vol. 3, pp. 246-252, 2011.

[19] H. Shareef, A. Mohamed, and A. A. Ibrahim, "Identification of voltage sag source location using $S$ and TT transformed disturbance power," Journal of Central South University, vol. 20, no. 1, pp. 83-97, 2013.

[20] C. R. Pinnegar and L. Mansinha, "A method of time-time analysis: The TT-transform," Digital Signal Processing, vol. 13, no. 4, pp. 588-603, 2003.

[21] C. R. Pinnegar, “Generalizing the TT-transform," Digital Signal Processing, vol. 19, no. 1, pp. 144-152, 2009.

[22] G.-Z. Shao, G. P. Tsoflias, and C.-J. Li, "Detection of near-surface cavities by generalized S-transform of Rayleigh waves," Journal of Applied Geophysics, vol. 129, pp. 53-65, 2016.

[23] M. V. Reddy and R. Sodhi, "A rule-based S-Transform and AdaBoost based approach for power quality assessment," Electric Power Systems Research, vol. 134, pp. 66-79, 2016.

[24] S. Zhang, P. Li, L. Zhang, H. Li, W. Jiang, and Y. Hu, "Modified S transform and ELM algorithms and their applications in power quality analysis," Neurocomputing, vol. 185, pp. 231-241, 2016.

[25] Z. Huang, J. Zhang, T. Zhao, and Y. Sun, "Synchrosqueezing S-transform and its application in seismic spectral decomposition," IEEE Transactions on Geoscience and Remote Sensing, vol. 54, no. 2, pp. 817-825, 2016.

[26] Y. Qin and L. Tian, "Pattern recognition and time location of power quality disturbances using TT-transform," in Proceedings of the 2010 International Conference on Intelligent System Design and Engineering Application, ISDEA 2010, pp. 53-56, China, October 2010.

[27] C. Simon, M. Schimmel, and J. J. Dañobeitia, "On the TTtransform and its diagonal elements," IEEE Transactions on Signal Processing, vol. 56, no. 11, pp. 5709-5713, 2008. 


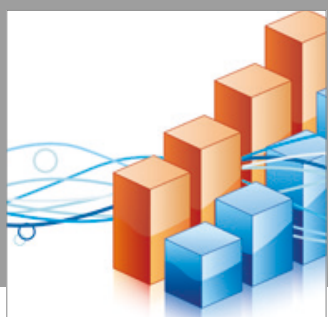

Advances in

Operations Research

vatersals

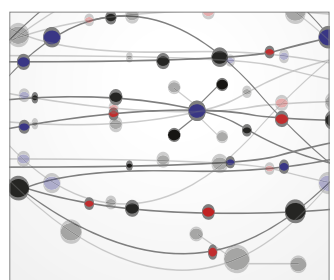

\section{The Scientific} World Journal
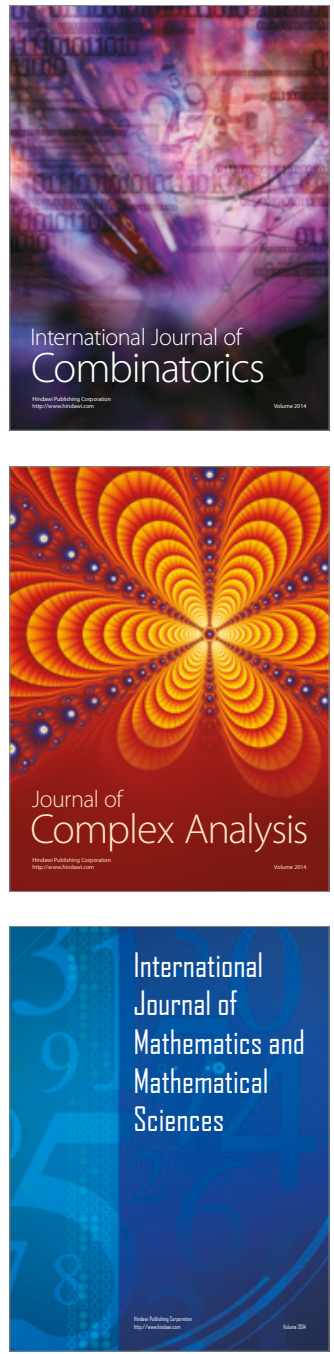
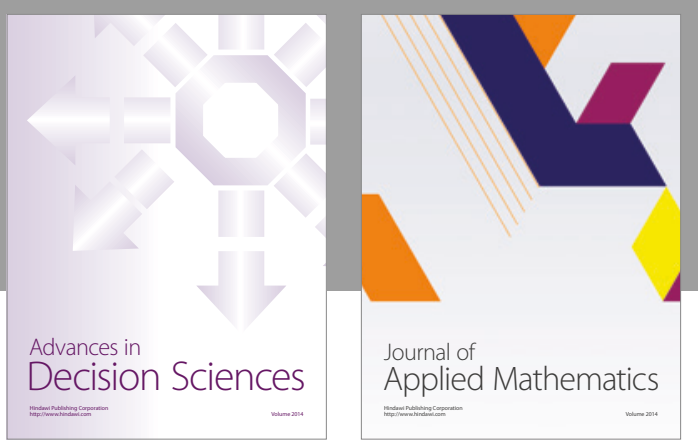

Algebra

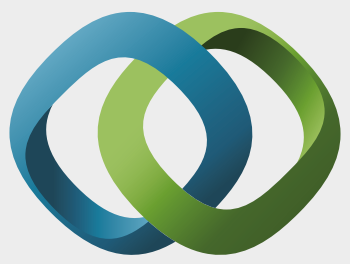

\section{Hindawi}

Submit your manuscripts at

https://www.hindawi.com
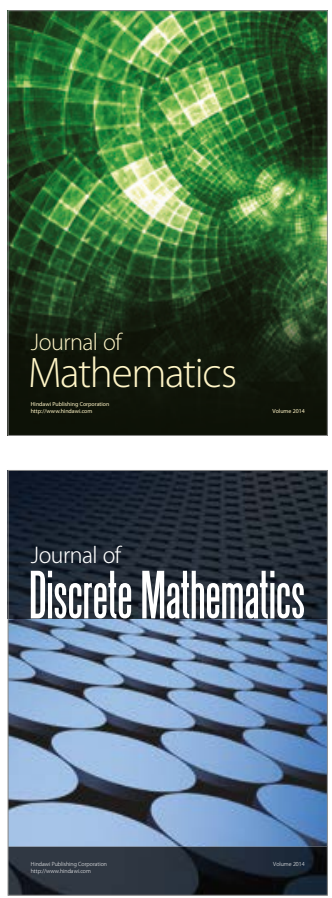

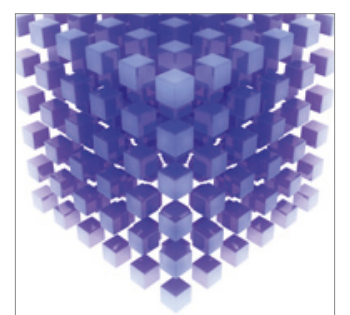

Mathematical Problems in Engineering
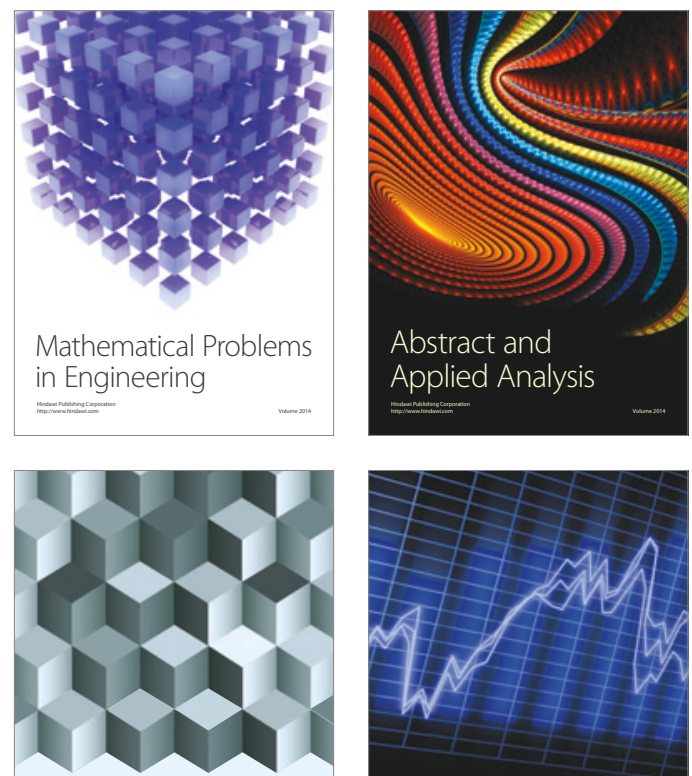

Journal of

Function Spaces

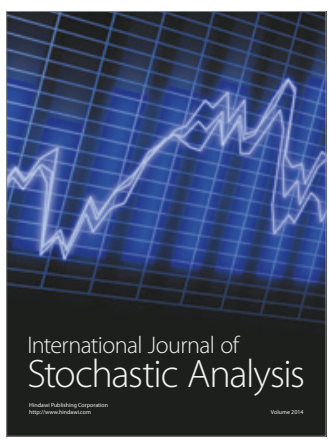

Probability and Statistics
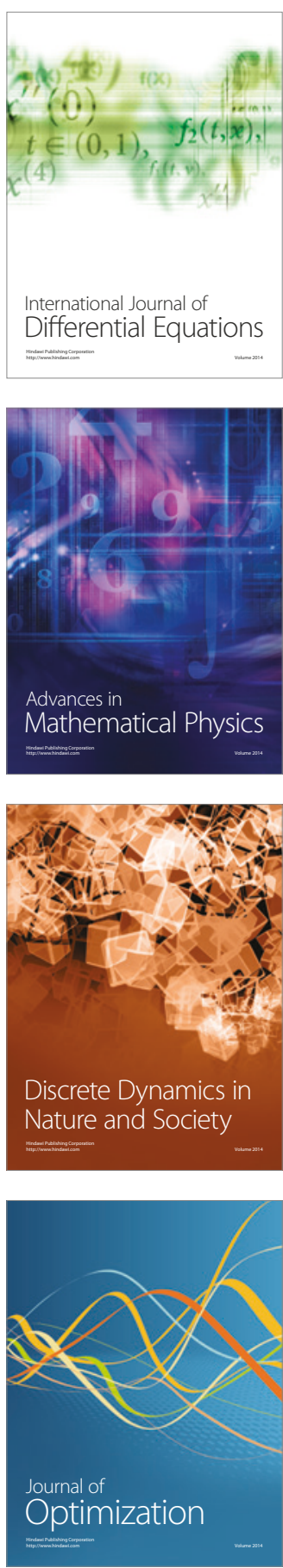